\title{
DAL TESTO ALLE PAROLE: STUDI CONTRASTIVI
}

\author{
ELIZAVETA KHACHATURYAN \\ Università di Oslo
}

\section{IN T R O D U ZIONE}

Negli ultimi decenni gli studi contrastivi continuano ad espandere il loro dominio segnando così l'inizio di una nuova fase: dall'analisi dei fenomeni grammaticali - prevalente negli studi del secolo scorso - l'interesse dei ricercatori si sta rivolgendo ora sempre di più verso le differenze comunicative che caratterizzano le lingue e riguardano diversi livelli linguistici, dalle strutture morfosintattiche fino alle proprietà di carattere sociolinguistico e pragmatico. ${ }^{1}$ Il punto di partenza di uno studio contrastivo diventano pertanto il testo ${ }^{2}$ visto come realizzazione generale di un qualsiasi atto comunicativo e i mezzi linguistici usati per costruirlo. Così (come vedremo nelle sezioni successive) l'analisi si concentra sulle forme usate in un contesto specifico o in una situazione comunicativa prestabilita.

Il presente articolo ha molteplici obiettivi. Oltre a introdurre i contributi del volume, esso si propone di descrivere il testo come oggetto di uno studio contrastivo individuando gli elementi importanti per la sua costruzione; e infine suggerisce possibili strade da seguire nei futuri studi italo-norvegesi, al momento ancora poco numerosi.

L'articolo è diviso in tre parti: la presentazione del testo come materiale per l'analisi contrastiva nella prima sezione sarà seguita, nella seconda sezione, dalla descrizione dei due tipi di analisi effettuata su due livelli (la struttura testuale e le parole usate), e si concluderà nella terza sezione con la presentazione dei contributi di questo volume.

\section{[1] IL TESTO COME OGgETTO DI STUDIO}

I manuali di linguistica definiscono il testo come "una produzione linguistica creata con l'intenzione e con l'effetto di trasmettere un particolare contenuto co-

[1] Un contributo importante a questa espansione dell'oggetto degli studi contrastivi è stato dato dai corpora, che hanno offerto maggiori possibilità per l'analisi dei testi di vari stili, permettendo anche di effettuare una ricerca veloce delle forme o strutture studiate. Per un esempio di vari tipi di studi basati sull'uso dei corpora (v. p.es. OSLa, 2014).

[2] In base alla forma della produzione (scritta oppure orale) si può distinguere tra testo e discorso. Di solito il testo ha la forma scritta. Questa proprietà impone alla struttura del testo certe caratteristiche come, per esempio, lo svolgimento lineare, l'impossibilità di retrocedere (a meno che non sia marcato esplicitamente nel testo), e il carattere statico. Il discorso, al contrario, svolgendosi nella comunicazione orale, viene considerato una produzione linguistica dinamica con possibilità di retroazione (v. p.es. Serianni 2003). I contributi del presente volume sono dedicati all'analisi del testo. 
municativo a uno o più destinatari" (Cerruti \& Cini 2007: 3). Nella costruzione del testo (di lunghezza illimitata, quindi da due parole fino a migliaia di pagine) sono coinvolte tre componenti indispensabili le cui caratteristiche sono importanti per la forma che assume il testo nel processo della costruzione: autore - messaggio lettore, dove l'autore (il mittente) si rivolge ai lettori (destinatari) con un certo messaggio comunicativo, la cui forma e il contenuto dipendono dalle caratteristiche dei partecipanti (autore-lettore) alla comunicazione. Tra queste caratteristiche bisogna distinguere i vari tipi di rapporti tra gli interlocutori, la situazione in cui si svolge la comunicazione, e anche la lingua usata nella comunicazione. Ogni tipo di testo prevede diverse forme di rapporti tra gli interlocutori (v. p.es. Cerruti \& Cini 2007, Palermo 2013, Serianni 2003). Inoltre, è noto che la coesione e la coerenza sono due requisiti importanti per creare un testo ben formato. La coesione, fondamentale per la struttura testuale, riguarda la forma e si realizza attraverso i vari modi in cui si collegano tra di loro le parti del testo. La coerenza è basata su una connessione logico-semantica tra le parti del testo e riguarda il contenuto e il significato del testo: sono le parole e le loro combinazioni ad avere un ruolo importante in questo caso.

Le regole che presiedono alla formazione di un testo sono universali ma i mezzi a disposizione per realizzarle variano a seconda della lingua. Questi mezzi riguardano, in primo luogo, la coesione (quindi la struttura) e la coerenza (quindi il contenuto: le parole e le espressioni usate per trasmettere il messaggio comunicativo) nel testo.

Un'analisi contrastiva può utilizzare come oggetto di studio i testi autentici e i testi costruiti in seguito a un esperimento linguistico. In questo secondo caso la costruzione del testo viene effettuata dagli informanti in base ai criteri prestabiliti in anticipo, prima di cominciare l'esperimento linguistico, il quale può prevedere diverse attività come, ad esempio, le descrizioni di immagini o di film, i giochi di ruolo (role plays) oppure le interviste. Come esempio di una tale costruzione del testo usato per l'analisi contrastiva possiamo citare uno studio danese (Korzen 2007, Skytte et al. 1999) dedicato al confronto della produzione linguistica e della strutturazione testuale in danese e in italiano. In questo studio gli informanti dovevano raccontare (alcuni per iscritto, gli altri oralmente) il contenuto di due film (muti) con Mr. Bean. Può servire da ulteriore esempio il progetto italo-australiano (Bettoni 2006) sulla realizzazione delle proteste. In questo caso l'analisi era focalizzata sulle differenze pragmatiche presenti nella comunicazione orale: gli informanti hanno partecipato ad un gioco di ruolo "recitando una serie di 9 scenette con diverse caratteristiche socio-pragmatiche" (Bettoni 2006: 121).

A seconda degli obiettivi dello studio, gli informanti coinvolti nell'esperimento possono costruire il testo sia nella loro madrelingua (L1) (come nello studio danese o nel progetto italo-australiano), sia nella lingua straniera (LS) che acqui- 
siscono. In questo secondo caso si discute spesso l'influenza della L1 sull'acquisizione della LS (p.es., Aijmer 2004, Fant \& Hancock 2015, Hasselgren 2002, Verhoeven \& Strömqvist 2001). A questo tema sarà dedicato l'ultimo studio del presente volume.

L'oggetto di uno studio contrastivo può essere costituito anche da testi tradotti messi a confronto con il testo originale oppure con un'altra traduzione in un'altra lingua, come illustrato dallo studio di Anne Flaen Anvik nel presente volume. $^{3}$

\section{[2] DUE APPROCCI ALL' ANALISI DEL TESTO}

Focalizzato sul testo come oggetto principale di analisi, lo studio contrastivo può svolgersi su due assi e descrivere: (i) le forme che fanno parte del testo, e (ii) il testo nel suo insieme come produzione finita. Sia le forme usate nel testo, sia la struttura del testo possono essere presentate in una prospettiva monolingue, che permetterà di stabilire le regole per questa lingua, e/o in una prospettiva plurilingue (prevista da un approccio contrastivo) che aiuterà a vedere (attraverso il confronto) le differenze tra i testi realizzati in diverse lingue.

\section{[2.1] Analisi della struttura testuale}

La struttura del testo non dipende solo dai partecipanti alla comunicazione. Certi aspetti della struttura testuale sono prescritti da regole che vigono a livello della lingua e che si dividono in: (i) regole di carattere intersegmentale (grammaticale e sintattico) che stabiliscono come connettere le varie parti della frase e (ii) regole di carattere soprasegmentale che descrivono come effettuare il collegamento tra le varie parti del testo (p.es. Andorno 2003, Cerruti \& Cini 2007, Palermo 2013, Serianni 2003). Tra i mezzi linguistici più diffusi e universali (ossia rilevanti per tutte le lingue) usati per creare un testo ben formato si possono citare i seguenti: elementi deittici, sostituzione lessicale (mediante sinonimi, iperonimi, nomi generali), riformulazione, ellissi, connettivi (che permettono di evitare le frasi nominali o le frasi giustapposte). ${ }^{4}$ Tutti questi strumenti, pur essendo universali, funzionano diversamente in vari tipi di testi, ${ }^{5}$ ma anche in varie lingue. Così ogni tipo di testo può essere caratterizzato in base alle strategie e fenomeni linguistici più tipici.

Per esempio, nei testi descrittivi (indipendentemente dalla lingua) sono spesso usati vari elementi locativi. Nei testi italiani di questo tipo prevalgono l'imperfetto e il verbo stare (Werlich 1975, in Coveri, Benucci \& Diadori 2005: 142-4 ).

[3] Va notato che anche le traduzioni nella stessa lingua della stessa opera, effettuate in periodi storici diversi e da traduttori diversi, possono servire da oggetto di studio interessante in quanto esempi rappresentativi dell'evoluzione della lingua e della società (v.p.es. Romanzi 2017).

[4] Per una descrizione dettagliata di questi elementi, si veda per esempio, i manuali di linguistica testuale (Andorno 2003, Cerruti \& Cini 2007, Palermo 2013, Serianni 2003).

[5] Per varie teorie sulla tipologia dei testi v. p.es. Coveri, Benucci \& Diadori 2005: 141-143 
Nei testi argomentativi è importante, ad esempio, l'uso dei connettivi (p.es. d'altronde, in effetti, pertanto, quindi). Un esempio di testo argomentativo è il testo accademico. È stato mostrato in alcuni studi (Clyne 1981, Gotti 2012, Hyland 1998, Siepmann 2006) che anche i testi accademici, nonostante una serie di proprietà abbastanza rigide e universali (se paragonati agli altri generi), possiedono alcune caratteristiche che variano da una lingua ad un'altra. Per esempio, Siepmann (2006) basandosi sugli studi precedenti e sulla propria analisi propone una tabella riassuntiva delle differenze stilistiche importanti per lo stile dei testi accademici scritti in inglese, francese e tedesco. Tra le differenze stilistiche individuate vengono descritti gli aspetti seguenti: relazione/ rapporto tra l'autore e il lettore, coerenza generale, struttura del testo, atti testuali, metalinguaggio, struttura del paragrafo, relazione logico-semantica insieme con l'uso dei segnali discorsivi, modalità testuale e hedging, autoreferenza dell'autore, nominazione degli agenti (2006: 142-3). Basandosi su questi aspetti, vari studi hanno dimostrato che il testo scritto in inglese ha una struttura lineare dove ogni paragrafo comincia con una frase principale (topic sentence) che viene sviluppata e conclusa alla fine del paragrafo (Siepmann 2006: 140).

Il testo scritto in francese invece dà più spazio a digressioni e riflessioni che non riguardano il soggetto in modo diretto. Inoltre, lo stile francese è molto più ornato (more ornate style), permette di usare metafore e costruzioni più espressive che in inglese non sono accettabili (Siepmann 2006). Queste differenze vengono spiegate dal fatto che anche la logica e la retorica si basano su alcuni principi tipici di una lingua e una cultura: "Logic (in the popular, rather than the logician's sense of the word) which is the basis of rhetoric, is evolved out of a culture; it is not universal. Rhetoric, then, is not universal either, but varies from culture to culture and even from time to time within a given culture" (Kaplan 1966/1980: 2).

Nello studio italo-danese (Korzen 2007) che analizza i testi descrittivi, sono state individuate alcune differenze che distinguono i testi costruiti in italiano dai testi in danese, tra le quali si possono citare a titolo d'esempio: l'uso più frequente dell'anafora "infedele" 6 in italiano che permette di evitare la ripetizione, la subordinazione in italiano usata come collegamento sintattico principale, invece della coordinazione più diffusa in danese. La struttura dei testi italiani e norvegesi non è oggetto di studio nel presente volume. Va notato, però, che è uno degli argomenti sul quale si possono focalizzare i futuri studi.

[6] L'anafora infedele, come viene definita in Korzen (2007), è un'anafora lessicalmente diversa dal suo antecedente, per esempio: Ho visto un'automobile nel nostro cortile ieri sera. Il veicolo era di una marca che non conosco. Oppure: Stasera arriva Umberto Eco. Lo scrittore italiano si ferma fino a domenica (gli esempi sono presi da Korzen 2007). 


\section{[2.2] Analisi delle parole usate}

Le parole e le espressioni usate nel testo sono importanti per garantire la coerenza del messaggio trasmesso. Questo tipo di connessione basato sulle conoscenze di carattere linguistico e culturale condivise dai partecipanti, garantisce l'accessibilità e la rilevanza dell'informazione per l'interlocutore (p.es. Andorno 2003, Cerruti \& Cini 2007, Palermo 2013, Serianni 2003). Le conoscenze di carattere linguistico riguardano il significato e le proprietà combinatorie delle parole (rapporti sintagmatici tra le parole). Di solito questa informazione fa parte delle regole della lingua e può essere appresa insieme con le altre regole durante il processo di acquisizione della lingua (sia della L1 sia della LS).

Le conoscenze di carattere culturale, trasmesse dalle parole, sono invece di molteplici tipi, possono contribuire alla costruzione del testo su vari livelli e sono studiate da diverse discipline. ${ }^{7}$ Per esempio, negli studi di Wierzbicka partendo dalle idee proposte da Humboldt, Sapir e Worf, viene descritta la specificità nazionale di ogni lingua (Wierzbicka 1992) e il carattere etnocentrico (ovvero l'orientamento verso la cultura del popolo) contenuto nel significato di alcune parole. Così attraverso l'analisi di alcune parole e costruzioni etnocentriche diventa possibile, secondo Wierzbicka, descrivere il carattere nazionale del popolo. Per esempio, nella cultura russa ci sono tre nozioni importanti che si ripetono spesso sia nella lingua quotidiana sia nella letteratura e non hanno un equivalente esatto nelle altre lingue: dusha (animo/anima), sud'ba (destino/sorte), toska (nostalgia/rimpianto) (Wierzbicka 1992). Lo stesso si potrebbe dire, per esempio, della parola fado in portoghese (Santos 2015). Negli studi di C. Kramsh dedicati alla didattica della lingua straniera (Kramsch 1993, 2006), con particolare riferimento agli approcci culturale e stilistico nell'insegnamento, si analizzano, per esempio, le differenze tra le associazioni (e/o i legami culturali) suscitate dalle parole nei parlanti di madrelingua diversa:

"When speaking of education (Bildung), German speakers do not think in terms of challenges or opportunities - two untranslatable words - but in terms of requirements (Anfroderungen) and performance (Leistung). (...) it raises the problem of wanting to express one world view through the language normally used to express another society's world view."

(Kramsch 1993: 20)

Certe conoscenze culturali uniscono tutti i parlanti della stessa lingua e fanno parte, secondo Kramsch (2006), della competenza simbolica (symbolic competence) del parlante. Proprio questa competenza è indispensabile per la comunicazione nella società moderna, dove gli obiettivi comunicativi vanno oltre a uno scambio

[7] Per una breve panoramica si veda p.es. Khachaturyan 2015. 
di informazioni prevedendo anche la manipolazione dei sistemi simbolici: "Communication itself has also expanded its goals from merely instrumental (problemsolving and exchange of information) to a much more sophisticated competence in the manipulation of symbolic systems" (Kramsch 2006: 251). Le conoscenze culturali e la competenza simbolica sono spesso difficili da acquisire: non sono presenti né nei dizionari, né nei manuali di lingua; inoltre, sono spesso dipendenti dalla figura del parlante (ovvero delle sue proprietà sociolinguistiche, come per esempio, età, studi eseguiti, professione) e dalle sue conoscenze (e/o esperienza). Allo stesso tempo si potrebbe dire che proprio le conoscenze di carattere culturale predefiniscono spesso (dal punto di vista del contenuto e della forma) quello che diciamo in varie situazioni.

Diversi studi contrastivi descrivono le differenze tra i parlanti nella stessa situazione comunicativa, ma di madrelingua diversa. Per esempio, a seconda della lingua cambia il modo di esprimere le emozioni (Pavlenko 2006), il modo di reagire ai complimenti (Alfonzetti 2013), o il modo di esprimere la protesta e la critica (Bettoni 2006, Bettoni \& Rubino 2007). Gli studi di questo tipo sono spesso chiamati di "pragmatica contrastiva" e di "pragmatica interculturale" (Bettoni 2006: 92-93).

Un altro gruppo di parole il cui significato è collegato con le conoscenze culturali è costituito dalle parole-realia, ossia "le parole che denotano cose materiali culturospecifiche" (Osimo 2010:157). Queste parole sono analizzate nell'ambito degli studi traduttivi (p.es. Osimo 2010) dove diversi approcci e strategie sono state elaborate per tradurre e classificare le parole-realia (per una descrizione dettagliata v. il contributo di Helene Johansen in questo volume).

Le parole usate in diversi tipi di testi, interpretabili non solo dal punto di vista linguistico ma anche portatrici di una certa informazione culturale sono oggetto degli studi presentati in questo volume.

\section{[3] IL CONTENUTO DEL VOLUME}

I contributi nel presente volume analizzano le parole che possono essere chiamate "parole con un contenuto culturale". Ogni articolo rappresenta un case-study e offre una descrizione dettagliata delle differenze linguistico-culturali tra le due lingue; ognuno di essi può servire da punto di partenza per un nuovo studio.

Tre articoli sono basati sull'analisi delle traduzioni. Il contributo di Anne Flaen Anvik descrive due traduzioni (una in norvegese e l'altra in italiano) delle favole di Hans Christian Andersen a partire dall'originale in danese. L'articolo di Helene Johansen è dedicato alle parole-realia usate in un libro per bambini della scrittrice norvegese Maria Parr e tradotto in italiano. Il contributo di Pernille Thull mette a confronto la traduzione in norvegese di due romanzi italiani (di Andrea Camilleri e di Simonetta Agnello Hornby rispettivamente). Tuttavia, il problema della traduzione viene affrontato secondo una prospettiva diversa in ogni studio pro- 
posto. L'articolo di Anne Flaen Anvik dimostra come l'espressione di alcuni atti linguistici convenzionali cambi a seconda della lingua riflettendo sulle differenze di carattere pragmatico-culturale tra le lingue: non basta sapere il significato della parola per usarla correttamente. L'articolo di Helene Johansen dedicato alle parole-realia si interroga sull'immagine di una realtà diversa creata attraverso le parole tipiche di una cultura ed inesistenti in un'altra, ossia su come trattare queste parole: mantenere e spiegare i fenomeni sconosciuti oppure sostituire e riformulare con espressioni più chiare, ma meno colorite? L'analisi di Pernille Thull descrive due atteggiamenti diversi nei confronti della presenza di un altro codice linguistico nel testo: le parole in dialetto siciliano usate nei due romanzi degli scrittori siciliani - Camilleri e Agnello Hornby - hanno funzioni diverse, così come diverse sono le strategie scelte nelle loro traduzioni per trasmettere questo rapporto con il dialetto in ciascuno di due scrittori.

L'articolo di Marit Viggen è dedicato al discorso politico in italiano e in norvegese: l'analisi prende in considerazione le proprietà linguistiche, concentrandosi in particolare sul lessico usato dai due politici di cui sono stati analizzati i discorsi e su alcune strutture grammaticali in essi contenute. Come viene dimostrato, alcune differenze sono imposte dalla struttura della lingua, altre dalle preferenze personali del parlante e del destinatario (ovvero del popolo nel caso dei discorsi presi in esame). Nello studio di Margrethe Førre Nardocci vengono descritte le parole e le combinazioni di parole al di fuori del testo. Quest'analisi permette di vedere il legame che esiste tra lingua e cultura, dimostrando come la cultura si rifletta e si codifichi nella lingua.

Il contributo di Elizaveta Khachaturyan offre invece un approccio diverso descrivendo i risultati di un esperimento condotto con due gruppi di apprendenti di italiano (L2) appartenenti a due madrelingue diverse (norvegese e russo). Questo studio è basato sull'ipotesi che gli strumenti linguistici a disposizione del parlante possano influenzare la costruzione del testo nel suo insieme.

Come vedremo, negli studi presentati nel volume l'analisi del testo costituisce il punto di partenza necessario per arrivare, attraverso un confronto dettagliato, effettuato su vari livelli linguistici, a nuovi e stimolanti risultati nel campo contrastivo per le lingue italiana e norvegese.

\section{RIF ERIMENTI BIBLIOGRAFICI}

Aijmer, K. 2004. Pragmatic markers in spoken interlanguage. Nordic Journal of English Studies 3(1).

Alfonzetti, G. 2013. Compliments. In M. Sbisà \& K. Turner (cur.), Pragmatics of Speech Actions (Handbooks of Pragmatics). De Gruyter Mouton.

Andorno, C. 2003. Linguistica testuale. Un'introduzione. Roma: Carocci.

Bettoni, C. 2006. Usare un'altra lingua. Guida alla pragmatica interculturale. Editori

Laterza. 
Bettoni, C. \& A Rubino. 2007. Reacting to complaints cross-culturally. Studi Italiani di Linguistica Teorica e Applicata (36). 483-498.

Cerruti, M. \& M. Cini. 2007. Introduzione elementare alla scrittura accademica. Editori Laterza.

Clyne, M. 1981. Culture and Discourse Structure. Journal of Pragmatics (5). 61-66.

Coveri, L., A. Benucci \& P. Diadori. 2005. Le varietà dell'italiano. Manuale di sociolinguistica italiana. Bonacci, Università per Stranieri di Siena.

Fant, L. \& V. Hancock. 2015. Marqueurs discursifs connectifs chez des locuteurs de L2 très avancés. In M. Borreguero Zuloaga \& S. Gómez-Jordana Ferary (cur.), Marqueurs du discours dans les langues romanes: une approche contrastive, 317-335. Limoges: Lambert Lucas.

Gotti, M. 2012. Cross-cultural aspects of academic discourse. Brno Studies in English 38(2). 59-78.

Hasselgren, A. 2002. Learner corpora and language testing: smallwords as markers of learners fluency. In S. Pons Bordería (cur.), Models of Discourse Segmentation. Explorations across Romance Languages. (Pragmatics and Beyond New Series), 185-218. Amsterdam: John Benjamins.

Hyland, K. 1998. Persuasion and Context. The Pragmatics of Academic Metadiscourse. Journal of Pragmatics (30). 437-455.

Kaplan, R. B. 1966/1980. Cultural Thought Patterns in Inter-Cultural Education. Language Learning XVI(1-20).

Khachaturyan, E. 2015. Shaping cultural identity through language: various types of cultural content in textbooks. In E. Khachaturyan (cur.), Language - Nation Identity: the "questione della lingua" in an Italian and non-Italian context. Cambridge Scholars Publishing.

Korzen, I. 2007. Mr. Bean e la lingua testuale. Considerazioni tipologico-comparative sulle lingue romanze e germaniche. In M. Barbera, E. Corino \& C. Onesti (cur.), Corpora e linguistica in rete, 209-224. Perugia: Guerra Edizione.

Kramsch, C. 1993. Language and Culture. Oxford University Press.

Kramsch, C. 2006. From communicative competence to symbolic competence. Modern Language Journal 90(2). 249-252.

Oksefjell Ebeling, S., A. Grønn, K. Rå Hauge \& D. Santos (cur.). 2014. OSLa: Oslo Studies in Language 6.1: Corpus-based Studies in Contrastive Linguistics.

Osimo, B. 2010. Propedeutica della traduzione. Hoepli.

Palermo, M. 2013. Linguistica testuale dell' italiano. Bologna: Il Mulino.

Pavlenko, A. (cur.). 2006. Bilingual minds: emotional experience, expression, and representation. Clevedon, UK: Multilingual Matters.

Romanzi, A. 2017. Il linguaggio di Holden Caulfield: una ritraduzione per trasformare il protagonista de Il Giovane Holden di Salinger. Una prima riflessione su ripetizioni e volgarismi. In V. Nigrisoli Wärnhjelm, A. Aresti, G. Colella \& M. Gargiulo (cur.), Edito, inedito, riedito: saggi dall'XI Congresso degli italianisti scandi- 
navi, Università del Dalarna, Falun, 9-11 giugno 2016, 261-271. Pisa: Pisa University Press.

Santos, D. 2015. Portuguese language identity in the world: adventures and misadventures of an international language. In E. Khachaturyan (cur.), LanguageNation-Identity: the "Questione della Lingua" in an Italian and Non-Italian Context. 31-54. Cambridge Scholars Publishing.

Serianni, L. 2003. Italiani scritti. Bologna: Il Mulino.

Siepmann, D. 2006. Academic Writing and Culture : An Overview of Differences between English, French and German. Meta: journal des traducteurs 51(1). 131150.

Skytte, G., I. Korzen, P. Polito \& E. Strudsholm (cur.). 1999. Tekststrukturering på italiensk og dansk. Resultater af en komparativ undersøgelse. København.

Verhoeven, L. \& S. Strömqvist (cur.). 2001. Narrative Development in a Multilingual Context. Amsterdam/Philadelphia: Benjamins.

Wierzbicka, A. 1992. Semantics, culture, and cognition: universal human concepts in culture-specific configurations. Oxford University Press.

AUTHOR CONTACT INFORMATION

Elizaveta Khachaturyan

Università di Oslo

elizaveta.khachaturyan@ilos.uio.no 